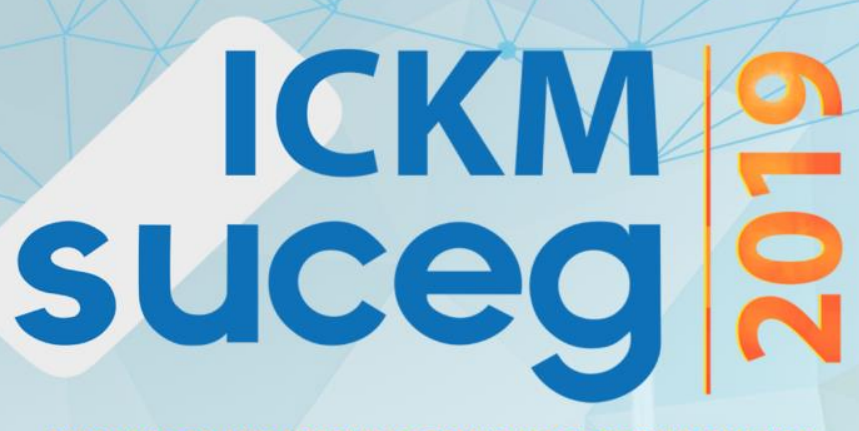

\title{
O ESTADO DA ARTE DO RELACIONAMENTO ENTRE COMPARTILHAMENTO DE CONHECIMENTO EM CADEIAS DE SUPRIMENTOS, INOVAÇÃO E DESEMPENHO DE ENTREGA
}

\author{
JUREMA SUELY DE ARAÚJO NERY RIBEIRO \\ Doutor, Universidade FUMEC, Universidade UEMG e Universidade PUC Minas \\ https://orcid.org/0000-0002-6465-6020, jurema.nery@gmail.com \\ FÁBIO CORRÊA \\ Doutor, Universidade FUMEC \\ http://orcid.org/0000-0002-2346-0187, fabiocontact@ gmail.com \\ RENATA DE SOUZA FRANÇA \\ Doutoranda, Universidade FUMEC e Universidade UEMG \\ https://orcid.org/0000-0002-3809-0975, profrenatafranca@gmail.com \\ ERIC DE PAULA FERREIRA \\ Doutorando, Universidade FUMEC \\ https://orcid.org/0000-0002-7513-501X, eric.p.f@gmail.com \\ FABRÍCIO ZIVIANI \\ Doutor, Universidade FUMEC, Universidade UEMG e Fundação Dom Cabral, \\ https://orcid.org/0000-0002-2705-846X, fabricio.ziviani@fumec.br
}

\begin{abstract}
RESUMO
Objetivo: Procurando entender como o relacionamento entre compartilhamento de conhecimento em cadeias de suprimentos, inovação e desempenho de entrega vem sendo tratados na literatura, este estudo objetivou apresentar uma revisão bibliométrica no intuito de responder a seguinte questão de pesquisa: como se apresenta o estado da arte do relacionamento entre compartilhamento de conhecimento em cadeias de suprimentos, inovação e desempenho de entrega?

Design/Metodologia/Abordagem: Pesquisa com abordagem quantitativa e qualitava, descritiva quanto ao tipo de estudo, bibliográfica e bibliométrica quanto aos meios.
\end{abstract}


Resultados: Este estudo possibilitou uma visão panorâmica dos trabalhos publicados acerca dos relacionamentos entre temáticas pesquisadas, indicando ser uma tendência de pesquisa no meio acadêmico e organizacional. Isto decorre dos resultados e oportunidades que podem ser proporcionados pelos relacionamentos articulados entre os construtos compartilhamento de conhecimento, cadeia de suprimentos, inovação e desempenho de entrega, a fim de aumentar a competitividade e alavancar o desempenho das cadeias de suprimentos.

Limitações da pesquisa: O pequeno número de trabalhos encontrados nas bases científicas, restringiu a amostragem, reduzindo a amplitude da pesquisa, sendo a sua principal limitação.

Originalidade/valor: Por meio das análises realizadas por essa pesquisa, percebeu-se que há reconhecimento das temáticas supracitadas como fontes necessárias de estudo, denotando a importância da realização mais de pesquisas, contudo poucos estudos realizam análise bibliométrica, ensejando a importância desta pesquisa.

Palavras-chave: Bibliometria. Cadeia de suprimentos. Compartilhamento de conhecimento. Desempenho de entrega. Inovação.

\title{
STATE OF THE ART OF RELATIONSHIP SHARING KNOWLEDGE IN SUPPLY CHAINS, INNOVATION AND DELIVERY PERFORMANCE
}

\begin{abstract}
Goal: Seeking to understand how the relationship between supply chain knowledge sharing, innovation and delivery performance has been treated in the literature, this study aimed to present a bibliometric review in order to answer the following research question: how is the state of the art of relationship between supply chain knowledge sharing, innovation and delivery performance?

Design/Methodology/Approach: Research with quantitative approach and qualitative, descriptive as to the type of study, bibliographic and bibliometric as to the means.

Results: This study provided a panoramic view of the published works on the relationships between researched themes, indicating that it is a research trend in the academic and organizational environments. This stems from the results and opportunities that can be provided by the articulated relationships between the knowledge sharing, supply chain, innovation, and delivery performance constructs in order to enhance competitiveness and leverage supply chain performance.
\end{abstract}

Limitations of the research: The small number of studies found in the scientific bases restricted sampling, reducing the scope of research, being its main limitation.

Originality/value: Through the analyzes performed by this research, it was realized that there is recognition of the above mentioned themes as necessary sources of study, denoting the importance of conducting more research, however few studies perform bibliometric analysis, giving rise to the importance of this research.

Keywords: Bibliometrics. Delivery performance. Innovation. Knowledge sharing. Supply chain. 


\section{INTRODUÇÃO}

A incessante busca por um crescimento lucrativo e sustentável tem despertado a necessidade de um melhor desempenho das organizações e entre organizações. No mercado atual, à medida que a concorrência ocorre nas cadeias de suprimentos, e a ênfase é dada para atender às demandas em constante mudança dos usuários finais, as interrupções na cadeia de suprimentos afetam o desempenho organizacional (Ho, Zheng, Yildiz \& Talluri, 2015). Estas interrupções podem ser causadas, dentre outros motivos, pelo aumento dos níveis de incerteza e a múltiplos eventos impactantes (Tse, Matthews, Tan, Sato \& Pongpanich, 2016).

O desempenho das cadeias pode estar relacionado, entre outros fatores, à forma como são estabelecidas as relações e práticas de compartilhamento de conhecimento com seus fornecedores (Castañeda, Pardo, \& Toulson 2015), aos processos de inovação empreendidos (Teixeira \& Valentim, 2017) alinhados aos requisitos dos clientes (Yan \& Azadegan, 2016). Contudo, cada vez mais, a competição ocorre não mais entre empresas, mas sim entre cadeias de fornecimento integradas (Byrne \& Heavey, 2010; Dyer, 2000). Desta forma, o Gerenciamento da Cadeia de Suprimentos ou Supply Chain Management, tem conquistado importante papel, tendo em vista a complexidade das atividades que desempenha e os desafios constantes que lhe são impostos, tais como a proeminente necessidade de obtenção de relações mais estreitas, versáteis e recíprocas entre clientes e fornecedores, a qual pode ser alcançada, dentre outras práticas, pelo compartilhamento de conhecimento entre os atores envolvidos (Hilsdorf, Rotondaro \& Pires, 2009).

Desta forma, o compartilhamento do conhecimento é um meio pelo qual o conhecimento se difunde nos níveis organizacionais e permite que esse ativo seja partilhado com indivíduos da organização, clientes, fornecedores, dentre outros stakeholders (Bem, Coelho \& Dandolini, 2016; Nonaka \& Takeuchi, 1997). As fontes da cadeia de suprimentos oferecem vantagens relacionadas à facilidade de transferência e implementação do conhecimento externo, no qual o conhecimento compreendido por ambas as partes pode ser facilmente transferido e aplicado (Yan et al., 2016). Fontes externas podem compartilhar ideias sobre novos componentes, novos mercados e novos processos com uma empresa, o que pode ajudar na comercialização do novo produto (Yan et al., 2016). Assim, a relevância do compartilhamento de conhecimento é imprescindível para as tratativas que visam prover melhoria na cadeia de suprimentos para entrega de valor aos clientes (Velu, 2014), podendo resultar em inovações e melhor desempenho econômico (de Sá Freire, Tosta, Helou Filho \& da Silva, 2012; Mintzberg, 1979). 
Entretanto, esse valor deixa de ser criado quando ocorrem interrupções e atrasos nas atividades de suprimentos da cadeia logística, pela ocorrência de falhas nos processos, na coordenação de atividades e na comunicação (Ribeiro, 2019). Essas falhas podem ser ocasionadas, entre outros fatores, pela falta de integração da cadeia de suprimentos e compartilhamento de conhecimentos entre fornecedores e clientes deficitário, interferindo negativamente no atendimento à demanda do cliente.

Diante da necessidade de entender como o relacionamento entre esses fatores vem sendo tratados na literatura e a importância de se analisar o relacionamento entre os construtos compartilhamento de conhecimento em cadeias de suprimentos, inovação e desempenho de entrega, este estudo objetivou apresentar uma revisão bibliométrica no intuito de responder a seguinte questão de pesquisas.

Desta análise, especificamente, pretende-se: i) identificar os estudos que tratam de compartilhamento de conhecimento em cadeias de suprimentos, inovação e desempenho de entrega; ii) apresentar as principais temáticas que têm sido abordadas nos estudos sobre compartilhamento de conhecimento em cadeias de suprimentos, inovação e desempenho de entrega, publicados nas bases científicas pesquisadas; iii) categorizar os estudos encontrados; iv) apontar os países com maior incidência de publicações sobre os construtos analisados e respectivas áreas de estudo; v) classificar as bases científicas mais profícuas.

A partir da definição da problemática a ser analisada e dos objetivos do presente estudo, fazse necessário compreender como a literatura vem tratando os construtos pesquisados, e também os locais onde estão sendo desenvolvidos estes estudos, delimitam-se as seguintes hipóteses: H1) existe um crescimento no número de publicações acerca dos constructos compartilhamento do conhecimento; cadeia de suprimentos, inovação e desempenho de entrega; H2) há predominância de publicações em determinados países.

Para dar seguimento a esta investigação, a pesquisa é subdividida em tópicos além desta introdução. Primeiramente (2.2), é explicitado a fundamentação teórica acerca dos construtos compartilhamento de conhecimento, cadeia de suprimentos, inovação e desempenho de entrega. Em sequência (2.3) são apresentados os procedimentos metodológicos utilizados para atingimento do objetivo almejado por esta pesquisa. Em continuidade (2.3), são apresentados os resultados e respectivas análises. Por conseguinte (3), as considerações finais são tecidas e as referências concluem esta pesquisa. 


\section{DESENVOLVIMENTO}

\subsection{REFERENCIAL TEÓRICO}

\subsubsection{Compartilhamento de conhecimento}

O compartilhamento de conhecimento pode ser entendido como compartilhamento de informações, ideias, experiências relevantes e sugestões do indivíduo com outros e entre organizações (Giannakis, 2008), bem como, "uma das etapas que subsidiam o conjunto de estratégias cujo objetivo é criar, disseminar e aplicar o conhecimento nos produtos, serviços e processos das organizações" (Duarte \& Silva 2017, p.5), sendo uma etapa do ciclo de criação do conhecimento (Nonaka \& Takeuchi, 1997).

Para Hung e Chuang (2009), é o núcleo de práticas de gestão, posto que o conhecimento, quando compartilhado, não desaparece, perde ou é depreciado; ao contrário, se eleva em contraponto a outros ativos convencionais (Sveiby, 1998). Para Duarte, Satur, Lira, Silva \& Lima, (2017, p.8) o compartilhamento do conhecimento está relacionado ao desenvolvimento de competências estratégicas, uma vez que "os novos conhecimentos e as competências oferecem à organização, novas alternativas e novos resultados".

Por meio da integração ou combinação de conhecimentos (Barney, 1991; Grant, 1996, Leonard-Barton, 1995; Prahalad \& Hamel, 1990) o compartilhamento do conhecimento ocorre na empresa e para empresas parceiras (Dyer et al., 2000). A habilidade da empresa em transferir suas melhores práticas (Szulanski, 1996) remete a um comportamento fundamental na criação e aplicação do conhecimento (Castañeda et al., 2015). Entretanto, a competência, tanto organizacional quanto individual, só se adquire com conhecimento viabilizado pelo processo de aprendizagem contínua (Duarte \& Santos, 2011).

Torna-se primordial, no entanto, que os esforços para disseminação do conhecimento estejam focados em garantir condições adequadas para ensejar esses processos internamente nas organizações e entre organizações, como uma forma de fortalecer as vantagens sustentáveis da rede de empresas. Estudiosos apontam a importância de se criarem mecanismos para que essa apropriação do conhecimento aconteça (Barbosa, 2008; Choo, 2003; Dalkir, 2011; Sveiby, 1998).

\subsubsection{Cadeia de suprimentos}

Empresas trabalhando em conjunto podem se tornar mais efetivas e eficientes ao encorajar a integração na cadeia de suprimentos e o compartilhamento de informações de maneira precisa e 
oportuna, resultando na otimização do fluxo de materiais ao longo da cadeia e eliminação de todos os processos que falham em agregar valor ao produto (Simatupang, Wright \& Sridharan 2002)

Algumas vantagens do gerenciamento integrado da cadeia de suprimentos são: produtos e serviços únicos, ciclos mais rápidos de Pesquisa e Desenvolvimento (P\&D), melhor qualidade dos produtos, competitividade em custo, ciclos menores de pedidos, flexibilidade às necessidades dos clientes, alavancagem no desempenho de entrega, melhor gerenciamento do ativo, maior velocidade do ciclo financeiro e relações recíprocas entre as empresas da cadeia (Croom, Fawcett, Osterhaus, Magnan, Brau \& McCarter, 2007).

Outras vantagens são percebidas, como níveis menores de estoque (implicando em riscos e custos mais baixos), aumento na produtividade, melhoria nos procedimentos gerenciais da empresa (aquisições, manufatura, distribuição, etc.), oportunidade de implementar processos padronizados e arquiteturas que compartilhem informações para alcançar o máximo desempenho da cadeia (Croom et al., 2007).

Para Yang, Yu, Liu \& Rui (2016) a efetividade da integração entre um grupo de organizações operando dentro de uma cadeia de suprimentos, poderia ser expressa em termos da qualidade e quantidade de conhecimento sendo trocado e a eficácia da coordenação. $O$ conhecimento mantido dentro de uma rede de parceiros é tão valioso quanto a capacidade da rede de transferir, processar e alavancá-lo (Kyläheiko, Jantunen, Puumalainen \& Luukka, 2011).

Um melhor gerenciamento da cadeia pressupõe uma maior integração entre os elos (atores) da cadeia e entre as atividades da própria organização, em cada elo individualmente (Croom et al., 2007). Essa gestão atua em prol do aumento da vantagem competitiva dos próprios atores, por meio da eficiência operacional, resultando na otimização da cadeia como um todo. Portanto, o desempenho de uma empresa será altamente influenciado por seus relacionamentos inter firmas ou alianças estratégicas (Dyer et al., 2000), para que assim se busque inovações.

\subsubsection{Inovação}

A inovação pode ser compreendida como uma ideia que, quando "incorporada no âmbito das organizações, em atividades produtivas, operacionais ou de gestão, propicia melhorias e/ou mudanças, em processos internos e nos resultados finais das operações organizacionais" (Barboza, Fonseca \& Ramalheiro, 2015, p.333), sendo que, a inovação no modelo de negócio envolve a 
descoberta e adoção de uma nova forma de disponibilizar, capturar e, ou, criar valor para uma empresa ou para o seu cliente (Velu, 2014).

A inovação é reconhecida como fator crítico para a sobrevivência e competitividade das organizações em seus ambientes, e também para a credibilidade das organizações não empresariais nos contextos dos meios sociais em que atuam (Barboza et al., 2015). Portanto, para sobreviver e competir as organizações devem inovar de maneira eficiente (Porter, 1989), desenvolvendo e dominando competências e habilidades (Jaruzelski et al., 2010).

Charterina, Landeta e Basterretxea (2018) afirmam que os conhecimentos advindos de compradores e fornecedores de uma empresa são fontes importantes para a identificação de desafios estratégicos e da inovação. Contudo, compradores e fornecedores que buscam melhorar sua capacidade inovadora com base em seu relacionamento, devem agir para compartilhar conhecimentos válidos para esse fim.

Isso remete a maximização dos recursos técnicos que facilitam a troca, frequência, conteúdo e qualidade do conhecimento (Charterina et al., 2018). Observa-se ainda que a participação dos fornecedores na inovação tecnológica tem um efeito positivo no desempenho operacional e no desempenho inovador (Johnsen, 2009).

Pode-se dizer também que a demanda do cliente é um importante antecedente da inovação colaborativa entre os intervenientes da cadeia e crucial para o design do produto e desenvolvimento de processos, de modo que, o envolvimento dos clientes com a inovação contribui positivamente, tanto para o desempenho da qualidade com para o desempenho da inovação (Kim, 2000).

Por fim, há um maior desempenho da cadeia, advindo de um atendimento mais expressivo das expectativas de entrega dos clientes pela integração da cadeia (Ribeiro, 2019).

\subsubsection{Desempenho de entrega}

O estudo do desempenho de entrega dentro de uma cadeia de suprimentos é fundamental, por isso a necessidade de medição e avaliação de desempenho na gestão da cadeia de suprimentos se torna imperativa (Gunasekaran \& Kobu, 2007; Guiffrida, 2014). A melhoraria do desempenho do processo de entrega é uma preocupação fundamental dos gerentes da cadeia de suprimentos e de logística (Forslund, Jonsson \& Mattsson, 2009).

Algumas variáveis podem influenciar no relacionamento e nos resultados de desempenho de entrega de uma cadeia de suprimentos tais como: adaptabilidade e abertura (Yang et al., 2016); 
confiança (Charterina et al., 2017); gestão estratégica de suprimentos (Revilla et al., 2015); capacidade de resposta (Kayakutlu \& Büyüközkan, 2010); competências (Kayakutlu et al., 2010); efetividade da comunicação (Yang et al., 2016); mecanismos de governança (Kyung, Liang \&Jung 2016); relacionamento (Yang et al., 2016).

Em síntese, o alcance de condições mais competitivas tem movido as organizações a desencadear ações expressivas em suas estruturas de forma a atuar com estratégias e ações gerenciais adequadas, a fim de oportunizar respostas às modificações necessárias, visando à sobrevivência no mercado, através do compartilhamento do conhecimento e inovação em prol de um atendimento de entrega superior do fornecedor.

\subsection{PROCEDIMENTOS METODOLÓGICOS}

Esta pesquisa apresenta um enfoque quantitativo e inferência qualitativa, em conformidade com a taxonomia proposta por Sampieri et al. (2006), pois baseia-se em métodos estatísticos de coleta e análise de dados para responder à questão de pesquisa.

Classifica-se como descritiva, quanto ao tipo de estudo, porque objetiva apresentar um panorama do estado da arte acerca do relacionamento entre compartilhamento de conhecimento, inovação e desempenho de entrega em cadeias de suprimentos, por meio da revisão bibliométrica dos artigos publicados nas bases científicas: Ebsco, Emerald, Scielo, Science Direct, Scopus, Spell.

Quanto aos meios, a pesquisa se classifica como bibliográfica e bibliométrica. De acordo com Vergara (2014) trata-se de pesquisa bibliográfica, pois utiliza a consulta a artigos publicados em bases científicas para obtenção de dados primários. Segundo Muniz Jr, Maia e Viola (2011) classifica-se como pesquisa bibliométrica, pois propõe a realização de uma análise crítica dos trabalhos científicos publicados acerca de um determinado assunto em um dado período de tempo, evidenciando os autores e os trabalhos mais relevantes.

Dessa forma, objetivando-se encontrar trabalhos na literatura que abordassem a temática estudada, no período de maio a julho de 2019, foi realizado o protocolo de revisão sistemática da literatura pesquisada exposto no Quadro 1.

Inicialmente após a efetivação da pesquisa foram obtidos 91 estudos, dos quais foram desconsiderados, de acordo com os critérios de exclusão um, dois e três, o total de 3, 9 e 48 publicações, respectivamente. Os 31 artigos resultantes foram lidos em sua totalidade, buscando 
identificar o estado da arte do relacionamento entre compartilhamento de conhecimento em cadeias de suprimentos, inovação e desempenho de entrega.

\section{Quadro 1: Protocolo de revisão sistemática da literatura.}

\begin{tabular}{|c|c|}
\hline Protocolo & Descrição \\
\hline $\begin{array}{l}\text { Quadro } \\
\text { conceitual }\end{array}$ & $\begin{array}{l}\text { O compartilhamento de conhecimentos, entre fornecedores e clientes, pode interferir no } \\
\text { atendimento à demanda de entrega ao cliente. Assim, busca-se identificar o estado da } \\
\text { arte do relacionamento compartilhamento de conhecimento em um contexto de cadeias } \\
\text { de suprimentos, considerando o desenvolvimento de um ambiente propício a inovação } \\
\text { para atingimento de um desempenho superior de entrega ao cliente. }\end{array}$ \\
\hline Contexto & $\begin{array}{l}\text { Compartilhamento de conhecimento em cadeias de suprimentos adotadas por empresas } \\
\text { de pequeno, médio e grande porte. }\end{array}$ \\
\hline Horizonte & Sem delimitação temporal. \\
\hline $\begin{array}{l}\text { Correntes } \\
\text { teóricas }\end{array}$ & $\begin{array}{l}\text { Especificamente, compartilhamento de conhecimento que tenham ocorrido em cadeias } \\
\text { de suprimentos, em ambiente propício a inovação, para atingimento de um desempenho } \\
\text { superior de entrega ao cliente. }\end{array}$ \\
\hline Línguas & Inglês \\
\hline $\begin{array}{l}\text { Critérios de } \\
\text { exclusão }\end{array}$ & $\begin{array}{l}\text { 1. Estudos duplicados; } \\
\text { 2. Estudos que não contemplem compartilhamento de conhecimento, inovação, } \\
\text { desempenho de entrega e que estejam fora do âmbito da cadeia de suprimentos; } \\
\text { 3. Tipos de documentos: article in press, book chapter, conference paper, conference } \\
\text { review e review. }\end{array}$ \\
\hline Descritores & $\begin{array}{l}\text { Termos presentes no título ou resumo ou palavra-chave com operador booleano "OR" e } \\
\text { operador booleano "AND" entre os termos: "knowledge management OR sharing } \\
\text { knowledge OR shared knowledge OR knowledge sharing" AND innovation AND supply } \\
\text { chain AND performance }\end{array}$ \\
\hline $\begin{array}{l}\text { Fontes } \\
\text { Pesquisadas }\end{array}$ & Ebsco, Emerald, Scielo, Science Direct, Scopus e Spell. \\
\hline
\end{tabular}

Fonte: Autores, adaptado de Dresch, Lacerda, Antunes Jr. (2015, p.142).

$\mathrm{Na}$ segunda fase, os 31 artigos foram categorizados considerando: dados dos autores, periódicos, países e áreas de atuação do estudo. A análise dos dados coletados foi realizada por meio do software Microsoft Office Excel. Os resultados foram expostos na seção 2.3.

\subsection{APRESENTAÇÃO E ANÁLISE DOS RESULTADOS}

Após a coleta dos dados, a próxima etapa promoveu a seleção e agrupamento das informações em comum entre os artigos encontrados. Os 31 artigos analisados, obtidos após a aplicação do protocolo de pesquisa, são relacionados no Quadro 2.

Com relação aos autores, apenas dois publicaram mais de um artigo, sendo Yang J. (artigo 9, 12 e 17 do Quadro 2) e Shamah R.A.M (artigo 8 e 13 do Quadro 2). 
Quadro 2 - Trabalhos relacionados e autores

\begin{tabular}{|c|c|c|c|}
\hline Id & Ano & Título & Autor \\
\hline 1 & 2005 & $\begin{array}{l}\text { Strategy of Relations among the Members of the Productive Chain } \\
\text { in Brazil: Reflections on the Theme }\end{array}$ & $\begin{array}{l}\text { Vasconcelos, M. C. R. L.; } \\
\text { Nascimento, R. M. E. }\end{array}$ \\
\hline 2 & 2007 & Knowledge management in 21st century manufacturing & Gunasekaran A.; Ngai E.W.T. \\
\hline 3 & 2007 & $\begin{array}{l}\text { Organizational learning, innovativeness, and organizational } \\
\text { performance: A qualitative investigation }\end{array}$ & $\begin{array}{l}\text { Yeung A.C.L.; Lai K.-H., Yee } \\
\text { R.W.Y. }\end{array}$ \\
\hline 4 & 2009 & $\begin{array}{l}\text { The effects of innovation-cost strategy, knowledge, and action in } \\
\text { the supply chain on firm performance }\end{array}$ & $\begin{array}{l}\text { Craighead C.W.; Hult G.T.M.; } \\
\text { Ketchen Jr. D.J. }\end{array}$ \\
\hline 5 & 2010 & $\begin{array}{l}\text { The impact of the supply chain on core competencies and } \\
\text { knowledge management: Directions for future research }\end{array}$ & $\begin{array}{l}\text { Halley A.; Nollet J.; Beaulieu } \\
\text { M.; Roy J.; Bigras Y. }\end{array}$ \\
\hline 6 & 2010 & Six tenets for developing an effective knowledge transfer strategy & McLaughlin S. \\
\hline 7 & 2010 & Effective supply value chain based on competence success & Gülgün K; Gülçin B. \\
\hline 8 & 2012 & Innovation within green service supply chains for a value creation & Shamah R.A.M. \\
\hline 9 & 2012 & $\begin{array}{l}\text { A structural model of supply chain performance in an emerging } \\
\text { economy }\end{array}$ & Yang J. \\
\hline 10 & 2013 & $\begin{array}{l}\text { Knowledge strategies for environmental innovations: the case of } \\
\text { Italian manufacturing firms }\end{array}$ & Marchi, V. D.; Grandinetti,R. \\
\hline 11 & 2014 & $\begin{array}{l}\text { Innovative knowledge sharing, supply chain integration and firm } \\
\text { performance of Australian manufacturing firms }\end{array}$ & Singh P.J.; Power D. \\
\hline 12 & 2015 & $\begin{array}{l}\text { The influence of environmental management practices and supply } \\
\text { chain integration on technological innovation performance- } \\
\text { Evidence from China's manufacturing industry }\end{array}$ & $\begin{array}{l}\text { Yang J.; Han Q.; Zhou J., Yuan } \\
\text { C. }\end{array}$ \\
\hline 13 & 2015 & $\begin{array}{l}\text { Facing the open innovation gap: measuring and building open } \\
\text { innovation in supply chains }\end{array}$ & $\begin{array}{l}\text { Shamah R.A.M.; Elssawabi } \\
\text { S.M. }\end{array}$ \\
\hline 14 & 2015 & $\begin{array}{l}\text { Building knowledge integration in buyer-supplier relationships: The } \\
\text { critical role of strategic supply management and trust }\end{array}$ & Revilla E.; Knoppen D. \\
\hline 15 & 2015 & $\begin{array}{l}\text { External knowledge acquisition and innovation: The role of supply } \\
\text { chain network-oriented flexibility and organisational awareness }\end{array}$ & Liao Y.; Marsillac E. \\
\hline 16 & 2016 & $\begin{array}{l}\text { Interorganizational alignment of strategic orientations in supply } \\
\text { chains }\end{array}$ & Abbade, E. B. \\
\hline 17 & 2016 & $\begin{array}{l}\text { Improving learning alliance performance for manufacturers: Does } \\
\text { knowledge sharing matter? }\end{array}$ & $\begin{array}{l}\text { Yang, J.; Guangsheng, Y; } \\
\text { Mingyu L.; Mingjie R. }\end{array}$ \\
\hline 18 & 2016 & $\begin{array}{l}\text { Impacts of buyer-supplier cooperation on trust and performance: } \\
\text { Moderating role of governance mechanism }\end{array}$ & Lee J.-S; Kim K.-T.; Hui L.. \\
\hline 19 & 2017 & $\begin{array}{l}\text { Mediation effects of trust and contracts on knowledge-sharing and } \\
\text { product innovation: Evidence from the European machine tool } \\
\text { industry }\end{array}$ & $\begin{array}{l}\text { Charterina, J.; Landeta, J; } \\
\text { Basterretxea, I. }\end{array}$ \\
\hline 20 & 2017 & $\begin{array}{l}\text { Comparing inter-organizational new product development } \\
\text { strategies: Buy or ally; Supply-chain or non-supply chain partners? }\end{array}$ & Tingting Y.; Arash A. \\
\hline 21 & 2017 & $\begin{array}{l}\text { Social innovation in the context of strategic knowledge } \\
\text { management processes for supply chain performance } \\
\text { enhancement }\end{array}$ & Jali M.N.; Abas Z.; Ariffin A.S. \\
\hline 22 & 2019 & $\begin{array}{l}\text { Leveraging customer knowledge to enhance process innovation: } \\
\text { Moderating effects from market dynaics }\end{array}$ & Nguyen, H., Harrison \\
\hline 23 & 2019 & Process network modularity, commonality, and greenhouse gas & Dooley, K.J.; Pathak, S.D.; \\
\hline
\end{tabular}




\begin{tabular}{|c|c|c|c|}
\hline & & emissions & $\begin{array}{l}\text { Kull, T.J.; Wu, Z.; Johnson, J.; } \\
\text { Rabinovich, E. }\end{array}$ \\
\hline 24 & 2019 & $\begin{array}{l}\text { The role of intellectual capital in new product development: Can it } \\
\text { become a liability? }\end{array}$ & $\begin{array}{l}\text { Subramanian, A.M.; Van de } \\
\text { Vrande, V. }\end{array}$ \\
\hline 25 & 2018 & $\begin{array}{l}\text { Impact of supply chain collaboration and knowledge sharing on } \\
\text { organizational outcomes in pharmaceutical industry of Bangladesh }\end{array}$ & Haque, M.; Islam, R., \\
\hline 26 & 2018 & $\begin{array}{l}\text { Exploring the role of intellectual capital in supply chain intelligence } \\
\text { integration }\end{array}$ & Shou, Y.; Hu, W.; Xu, Y \\
\hline 27 & 2017 & $\begin{array}{l}\text { The influence of socialisation and absorptive capacity on buyer's } \\
\text { innovation performance }\end{array}$ & $\begin{array}{l}\text { Chowdhury, M.; Jayaram, J.; } \\
\text { Prajogo, D. }\end{array}$ \\
\hline 28 & 2017 & $\begin{array}{l}\text { Organisational learning capability in SMEs: An empirical } \\
\text { development of innovation in the supply chain }\end{array}$ & $\begin{array}{l}\text { Thomas, A.; Dorrington, P.; } \\
\text { Costa, F.; Loudon, G.; } \\
\text { Francis, M.; Fisher, R. }\end{array}$ \\
\hline 29 & 2007 & $\begin{array}{l}\text { Losing Managerial Discretion: The Unexplored Risk of } \\
\text { Collaborative Information Sharing. }\end{array}$ & Fabian, F.; Dhillon, G. \\
\hline 30 & 2011 & $\begin{array}{l}\text { Investigation into Current Supply Chain Practices at a Private } \\
\text { Healthcare Provider in South Africa }\end{array}$ & $\begin{array}{l}\text { Bauernschmitt, E.; Conradie, } \\
\text { W. M. }\end{array}$ \\
\hline 31 & 2008 & Ireland's Innovation Performance: 1991 to 2005 & Hewitt-Dundas, N.; Roper, S. \\
\hline
\end{tabular}

Fonte: Dados da pesquisa (2019).

Ao considerar a totalidade de artigos publicados, percebeu-se uma inexpressividade na predominância de autoria, demonstrando que os construtos estão sendo pesquisados por autores diversos, não havendo um autor recorrente dentre as publicações.

Com o objetivo de agrupar os artigos publicados que tratam do mesmo tema, foram criadas categorias que emergiram da análise das publicações, sendo essas relacionadas no Quadro 3.

\section{Quadro 3- Conceitos das categorias utilizadas para agrupar os artigos estudados}

\begin{tabular}{|l|l|}
\hline \multicolumn{1}{|c|}{ Categorias } & \multicolumn{1}{c|}{ Síntese das Categorias } \\
\hline $\begin{array}{l}\text { Ferramentas de Gestão } \\
\text { de Canais de Distribuição }\end{array}$ & $\begin{array}{l}\text { Estudos referentes ao desenvolvimento de modelos e/ou práticas de gestão } \\
\text { que cooperem para agregação de valor ao longo da cadeia de distribuição. }\end{array}$ \\
\hline $\begin{array}{l}\text { Gestão Estratégica da } \\
\text { Cadeia de Suprimento }\end{array}$ & $\begin{array}{l}\text { Artigos cujo objeto de estudo consiste no fortalecimento das relações com os } \\
\text { fornecedores visando agregação de valor ao fluxo da cadeia. }\end{array}$ \\
\hline $\begin{array}{l}\text { Governança da Cadeia } \\
\text { de Suprimento }\end{array}$ & $\begin{array}{l}\text { Reúne os artigos que demonstrem as relações de poder que ocorrem ao } \\
\text { longo da cadeia, em que uma ou mais empresas coordenam e controlam as } \\
\text { atividades fundamentais de desenvolvimento de produtos e processos-chave. } \\
\text { Ou seja, refere-se ao exercício de legitimidade de um elemento na } \\
\text { coordenação de relação com outras empresas participantes do sistema. }\end{array}$ \\
\hline
\end{tabular}

Fonte: Elaborado pelos autores (2019).

A relação dos artigos analisados (Quadro 2) e das categorias emergentes da análise desses (Quadro 3), permitiu visualizar a proporção de artigos por categorias, considerando a classificação destes pela dispersão temporal percentílica das pesquisas, conforme Tabela 1. 
Tabela 1 - Proporção de artigos agrupados por categorias

\begin{tabular}{llllllllllllllll}
\hline \multicolumn{1}{c}{ Categoria/Ano } & \multicolumn{10}{c}{ Ano 2005 a 2019(\%) } \\
\cline { 2 - 10 } & 05 & 07 & 08 & 09 & 10 & 11 & 12 & 13 & 14 & 15 & 16 & 17 & 18 & 19 & $\%$ Total \\
\hline $\begin{array}{l}\text { Ferramentas de Gestão de } \\
\text { Canais de Distribuição }\end{array}$ & 0,0 & 3,2 & 3,2 & 3,2 & 0,0 & 0,0 & 6,5 & 0,0 & 3,2 & 12,9 & 0,0 & 6,5 & 6,5 & 9,7 & 54,9 \\
$\begin{array}{l}\text { Gestão Estratégica da } \\
\text { Cadeia de Suprimento }\end{array}$ & 3,2 & 3,2 & 0,0 & 0,0 & 6,5 & 0,0 & 0,0 & 3,2 & 0,0 & 0,0 & 3,2 & 3,2 & 0,0 & 0,0 & 22,6 \\
$\begin{array}{l}\text { Governança da Cadeia de } \\
\text { Suprimento }\end{array}$ & 0,0 & 3,2 & 0,0 & 0,0 & 3,2 & 3,2 & 0,0 & 0,0 & 0,0 & 0,0 & 6,5 & 3,2 & 3,2 & 0,0 & 22,6 \\
\hline
\end{tabular}

Fonte: Elaborado pelos autores (2019).

A categoria com maior representatividade foi Ferramentas de Gestão de Canais de Distribuição, com total de 54,9\%, sendo os anos de 2015 e 2019 os mais expressivos, com 12,9\% e 9,7\% dos artigos respectivamente. A categoria Gestão Estratégica da Cadeia de Suprimento e Governança da Cadeia de Suprimento apresentaram cada uma o total de 22,6\%, com ênfase no ano de 2016, para Governança da Cadeia de Suprimento com 6,5\% dos artigos.

Isto revela que a categoria Ferramentas de Gestão de Canais de Distribuição, com total de $54,9 \%$ dos artigos, tem seu objeto de estudo relacionado à temática desenvolvimento de modelos e/ou práticas de gestão visando agregação de valor ao fluxo da cadeia de suprimento. A categoria Gestão Estratégica da Cadeia de Suprimento com total de 22,6\% dos trabalhos publicados, exprime que as publicações que optaram por essa temática exploram fortalecimento das relações com os fornecedores visando agregação de valor ao fluxo da cadeia.

Já a categoria Governança da Cadeia de Suprimento, com total de 22,6\% dos artigos demonstra a preocupação as relações de poder que ocorrem ao longo da cadeia, em que uma ou mais empresas coordenam e controlam as atividades fundamentais de desenvolvimento de produtos e processos-chave.

O artigo mais antigo é do ano 2005 e, desde então, o número de publicações sobre o tema tem se elevado representativamente. Isto permite inferir que abordagens que relacionam o compartilhamento do conhecimento na cadeia de suprimentos junto a inovação são recentes. O ano de publicação dos artigos está compreendido no intervalo de 2005 a 2019 (Tabela 2) e agrupados em intervalos de cinco anos. 
Tabela 2 - Número de artigos publicados a cada cinco anos

\begin{tabular}{lcc}
\hline \multicolumn{1}{c}{ Período } & Total de artigos & Percentual de artigos \\
\hline Antes de 2004 & 0 & $0 \%$ \\
De 2005 a 2009 & 6 & $19 \%$ \\
De 2010 a 2014 & 8 & $26 \%$ \\
De 2015 a 2019 & 17 & $55 \%$ \\
\hline Total geral & 31 & $100 \%$ \\
\hline
\end{tabular}

Fonte: Dados da pesquisa (2019).

No período anterior a 2004 não há registro de publicações inerentes aos descritores aplicados nesta pesquisa, considerando as bases pesquisadas, conforme Tabela 2. Observando os dados acerca da quantidade de publicações feitas entre 2005-2017, foi possível perceber que o número cresceu significativamente a partir do ano 2010. De 2005-2009 houveram apenas seis publicações, que evoluíram para oito entre 2010-2014, chegando a 17 publicações em 2015-2019.

Neste período de 15 anos de publicação, pode-se observar que até o final do ano de 2014 10 anos de pesquisas - foram publicados $45 \%$ dos artigos selecionados. Em contrapartida nos últimos cinco anos (2015 a 2019) foram publicados 55\% dos artigos, o que mostra um crescimento do interesse sobre compartilhamento de conhecimento, inovação, cadeia de suprimentos e desempenho propostos neste estudo.

Com base nesse aumento do número de publicações, pode-se inferir que o interesse pelo relacionamento entre os construtos pesquisados se elevou, podendo ser um indício e uma consequência desse fato, comprovando a Hipótese 1 desta pesquisa. Vale ressaltar ainda que no ano de 2019 foram considerados apenas seis meses de publicações, contando com três publicações, quantidade semelhante ao número de publicação anual dos últimos seis anos.

No que tange à publicação por país, os que mais publicaram foram a China e os EUA, com seis publicações cada, seguidos pela Austrália com quatro publicações e pelo Reino Unido com três, conforme Tabela 3.

Os dados apresentados na Tabela 3 demonstram que os 31 artigos analisados foram desenvolvidos por doze países, sendo que quatro países assumem $61 \%$ dos artigos, ou seja, aproximadamente $38 \%$ dos artigos publicados foram pela China e Estados Unidos e 13\% Austrália e Reino Unido com 10 \%, demonstrando uma predominância significativa de estudos nestes países mencionados, fato que comprovou a Hipótese 2. 
Tabela 3 - Número de publicações por país

\begin{tabular}{lcc}
\hline \multicolumn{1}{c}{ País } & Número de publicações & Percentual publicações \\
\hline China & 6 & $19 \%$ \\
Estados Unidos & 6 & $19 \%$ \\
Austrália & 4 & $13 \%$ \\
Reino Unido & 3 & $10 \%$ \\
Brasil & 2 & $6 \%$ \\
Egito & 2 & $6 \%$ \\
Espanha & 2 & $6 \%$ \\
Malásia & 2 & $6 \%$ \\
Canadá & 1 & $3 \%$ \\
Coréia do sul & 1 & $3 \%$ \\
Itália & 1 & $3 \%$ \\
Turquia & 1 & $3 \%$ \\
\hline \multicolumn{1}{c}{ Total } & $\mathbf{3 1}$ & $\mathbf{1 0 0}$ \\
\hline \multicolumn{2}{c}{}
\end{tabular}

Fonte: Dados da pesquisa, 2019.

Essa predominante participação da China e dos Estados Unidos (Tabela 3) demonstrou a preocupação destes com o aperfeiçoamento da cadeia de suprimentos para obtenção de uma melhor performance e atendimento as expectativas de entrega dos clientes, por meio do compartilhamento de conhecimento e/ ou de processos de inovação. Em especial, os estudos realizados na China, segundo Yang et al. (2016) servem como um bom cenário para um estudo empírico sobre cadeia de suprimentos, devido a economia de transição emergente da China, ou seja, sua transição de planejamento central para concorrência no mercado. Este cenário atraiu um grande número de empresas interessadas em mover as suas operações de produção para a China, objetivando tirar proveito de seus baixos custos de recursos e mão-de-obra.

Em relação aos artigos publicados de acordo com a área de atuação de pesquisa, a Tabela 4 apresenta os quantitativos e percentis relacionados a essa perspectiva.

Tabela 4 - Número de artigos publicados de acordo com a área de atuação da pesquisa

\begin{tabular}{lcc}
\hline \multicolumn{1}{c}{ Área de atuação } & Número de artigos & Percentual de artigos \\
\hline Indústrias & 14 & $45 \%$ \\
Cadeia de Suprimentos & 16 & $52 \%$ \\
Hotéis & 1 & $3 \%$ \\
\hline \multicolumn{1}{c}{ Total } & $\mathbf{3 1}$ & $\mathbf{1 0 0 \%}$ \\
\hline
\end{tabular}

Fonte: Dados da pesquisa, 2019.

Com relação a área de atuação da pesquisa dos artigos em questão (Tabela 4), as áreas Indústria e Cadeia de suprimentos apresentaram $45 \%$ e $52 \%$ respectivamente dos artigos publicados no período analisado, com 30 do total de 31 artigos publicados. Este resultado demonstra um 
equilíbrio de interesses de estudo dos construtos pesquisados nas áreas de atuação Indústria e Cadeia de Suprimentos.

O número de artigos selecionado por base de dados Scopus, Ebsco, Emerald, Science Direct, Scielo, Spell, foi 22, 7, 4, 2, 1, 1 respectivamente, foi demonstrado na Tabela 5.

Tabela 5 - Número de artigos publicados por base científica

\begin{tabular}{lcc}
\hline \multicolumn{1}{c}{ Base científica } & Número de artigos & Percentual de artigos \\
\hline Scopus & 16 & $52 \%$ \\
Ebsco; Scopus & 4 & $13 \%$ \\
Ebsco & 3 & $10 \%$ \\
Emerald & 2 & $6 \%$ \\
Emerald; Scopus & 2 & $6 \%$ \\
Science Direct & 2 & $6 \%$ \\
Scielo & 1 & $3 \%$ \\
Spell $\quad$ Total & 1 & $3 \%$ \\
\hline \multicolumn{1}{c}{$\quad \mathbf{3 1}$} & $\mathbf{1 0 0 \%}$ \\
\hline \multicolumn{2}{c}{$\quad$}
\end{tabular}

Fonte: Dados da pesquisa, 2019.

A tabela 5 evidenciou a participação, quando considerado a publicação exclusiva de $52 \%$ (16 artigos) representada pela base científica Scopus, seguida pela Ebsco com 10\% (3 artigos). Por sua vez, a Emerald e a Science Direct publicaram cada uma 6\% dos artigos. Já a Scielo e a Spell participaram individualmente com 3\% do total de publicações. Foram encontrados seis trabalhos publicados em duas bases distintas, sendo 4 artigos simultaneamente pela Ebsco e Scopus, e 2 artigos pela Emerald e Scopus. A expressiva participação da Scopus denota a relevante contribuição deste periódico na investigação do estado da arte dos construtos aqui estudados.

Com relação ao tipo de abordagem de pesquisa utilizado nos estudos, observa-se que 18 pesquisas foram quantitativas e 13 qualitativas, denotando um predomínio de técnicas estatísticas para quantificar opiniões e informações ao estruturar dados, a fim de se obter conclusões gerais sobre os construtos pesquisados

Pode-se então inferir, por meio das análises realizadas por essa pesquisa, que há reconhecimento das temáticas supracitadas como fontes necessárias de estudo. Conforme Bowersox, Closs e Cooper (2014) e Flynn, Huo e Zaho (2010), o processo de intensificação nos relacionamentos estabelecidos entre organizações de uma cadeia, no intuito de alcançar vantagens competitivas por meio de fluxos eficazes e eficientes de produtos, serviços, informações e capital, agregam máximo de valor ao cliente final. Portanto, é uma temática que demanda novas pesquisas. 


\section{CONSIDERAÇÕES FINAIS}

Com base nos dados obtidos com a realização desse estudo, pode-se inferir que o relacionamento entre compartilhamento de conhecimento, cadeia de suprimentos, inovação e desempenho são temas relativamente recentes, uma vez que as publicações a esse respeito emergem, expressivamente (55\%), a partir de 2015, comprovando a Hipótese 1. Os temas receberam, gradativamente, atenção de gestores e pesquisadores, principalmente as áreas indústria e cadeia de suprimentos conforme verificado no estudo.

De acordo com os dados aferidos pôde-se verificar a comprovação da Hipótese 2, uma vez que foi identificado predominância de publicações nos países China e Estados Unidos, aproximadamente $38 \%$ dos artigos. Ao considerar a totalidade de artigos publicados, percebeu-se uma inexpressividade na predominância de autoria, demonstrando que os construtos estão sendo pesquisados por autores diversos.

A respeito dos tipos de abordagem empregadas pelos autores que publicaram sobre as temáticas investigadas neste estudo, nota-se uma predominância do método quantitativo. Com relação a área de atuação da pesquisa dos artigos em questão, as áreas indústria (45\%) e cadeia de suprimentos (52\%) abrangeram 97\% dos artigos publicados no período analisado acerca do assunto pesquisado, com 30 do total de 31 artigos publicados.

Faz-se importante ressaltar que os objetivos específicos i, ii, iii, iv e v foram atingidos sendo apresentados no estudo por Quadro 1 e 2, Tabela 4, Quadro 3 e Tabela 1, Tabela 3 e Tabela 5 respectivamente.

Por fim, este estudo possibilitou uma visão panorâmica dos trabalhos publicados acerca dos relacionamentos entre temáticas pesquisadas, indicando ser uma tendência de pesquisa no meio acadêmico e organizacional. Isto decorre dos resultados e oportunidades que podem ser proporcionados pelos relacionamentos articulados entre os construtos compartilhamento de conhecimento, cadeia de suprimentos, inovação e desempenho de entrega, a fim de aumentar a competitividade e alavancar o desempenho das cadeias de suprimentos, através da manutenção e ou desenvolvimento dos ativos intangíveis, podendo oferecer inúmeras vantagens às organizações pertencentes.

Entretanto, foi possível observar por meio desta pesquisa que existe um pequeno número de estudos realizados relacionando os construtos compartilhamento de conhecimento, inovação, cadeia de suprimentos e desempenho, fato este que denota a importância da realização de um número maior 
de pesquisas sobre a temática em questão, contudo poucos estudos realizam análises bibliométricas ensejando, assim, a importância desta pesquisa. Esse pequeno número de trabalhos encontrados nas bases científicas, restringiu a amostragem, reduzindo a amplitude da pesquisa, sendo a sua principal limitação.

\section{REFERÊNCIAS}

Barbosa, R. R. (2008). Gestão da informação e do conhecimento: origens, polêmicas e perspectivas. Informação \& Informação, Londrina, v. 13, n. esp., p. 1-25.

Barboza, R. A. B., Fonseca, S. A., \& Ramalheiro, G. C. F. (2015). Inovação em micro e pequenas empresas por meio do Serviço Brasileiro de Respostas Técnicas. Review of Administration and Innovation - RAI, v. 12, n. 3, p. 329-349.

Barney, J. (1991). Firm resources and sustained competitive advantage. Journal of Management, v. 17, n. 1, p. 99-120.

Bem, R. M. De, Coelho, C. C. De S. R., \& Dandolini, G. A. (2016). Knowledge management framework to the university libraries. Library Management, v. 37, n. 4/5, p. 221-236.

Bowersox, D. J., Closs, D. J., \& Cooper, M. (2014). Gestão Logística da Cadeia de Suprimentos. $4^{\mathrm{a}}$ Edição.

Byrne, P., \& Heavey, C. (2010). Simulation model of a vertically integrated supply chain: a case study. International Journal of Industrial Engineering: Theory, Applications and Practice, North America, 13, sep.

Castañeda, D., Pardo, C., \& Toulson, P. (2015). Knowledge sharing instrument validation: broader perspective for global organizations. Eletronic Journal of Knowledge Management, v. 13, 1, pp. 312.

Charterina, J., Landeta, J., \& Basterretxea, I. (2018). Mediation effects of trust and contracts on knowledge-sharing and product innovation: Evidence from the European machine tool industry. European Journal of Innovation Management, v. 21, n. 2, p. 274-293.

Choo, C. W. (2003). A organização do conhecimento: como as organizações usam a informação para criar significado e construir conhecimento e tomar decisões. São Paulo: SENAC.

Croom, S., Fawcett, S. E., Osterhaus, P., Magnan, G. M., Brau, J. C., \& McCarter, M. W. (2007). Information sharing and supply chain performance: the role of connectivity and willingness. Supply Chain Management: An International Journal. v.12, n.5, p.358.

Dalkir, K. (2011). Knowledge Management in Theory and Practice. Cambridge, London: The MIT Press. 
De Sá Freire, P., Tosta, K. C. B. T., Helou Filho, E. A., \& da Silva, G. G. (2012). Memória organizacional e seu papel na gestão do conhecimento. Revista de Ciências da Administração, 14(33), 41-51.

Dresch, A., Lacerda, D. P., \& Antunes, J. A. V. (2015). Design science research: método de pesquisa para avanço da ciência e tecnologia. Bookman Editora, 2015. 161 p.

Duarte, E. N., \& Santos, M. L. da C. (2011). O Conhecimento na administração estratégica. Perspectivas em Gestão \& Conhecimento, 1.1: 15-24.

Duarte, E. N., Satur, R. V., Lira, S. De Lu., Silva, N. B. X., \& Lima, E. S. de. (2017). Práxis de gestão do conhecimento no ambiente das organizações no escopo da Ciência da Informação. Anais... XVII Encontro Nacional de Pesquisa em Ciência da Informação (XVII ENANCIB).

Duarte, N. T. De O., \& Silva, A. K. A. da. (2017). O compartilhamento na perspectiva da gestão da informação e do conhecimento. Anais... XVII Encontro Nacional de Pesquisa em Ciência da Informação (XVII ENANCIB).

Dyer, J. H. (2000) Collaborative Advantage: Winning Through Extended Enterprise Supplier Networks. New York: Oxford University Press, 209 p.

Flynn, B. B., Huo, B., \& Zhao, X. (2010). The impact of supply chain integration on performance: A contingency and configuration approach. Journal of Operations Management, v. 28, n. 1, p. 5871.

Forslund, H., Jonsson, P., \& Mattsson, S. (2008). Order-to-delivery process performance in delivery scheduling environments. International Journal of Productivity and Performance Management, $\mathrm{v}$. 58 , n. 1, p. 41-53.

Giannakis, M. (2008). Facilitating learning and knowledge transfer through supplier development. Supply Chain Management: An International Journal, v. 13, n. 1, p. 62-72. Grant, R. M. (1996). Toward a knowledge-based theory of the firm. Strategic Management Journal, v. 17, n. 2, p. 109-122.

Guiffrida, A. L. (2014). Recent trends in supply chain delivery models. World Academy of Science, Engineering and Technology, International Journal of Social, Behavioral, Educational, Economic, Business and Industrial Engineering, v.8, n.6, p.1823-1826.

Gunasekaran, A., \& Kobu, B. (2007). Performance measures and metrics in logistics and supply chain management: a review of recent literature (1995-2004) for research and applications. International Journal of Production Research, v. 45, n. 12, p. 2819-2840.

Hilsdorf, W. De C., Rotondaro, R. G., \& Pires, S. R. I. (2009). Integração de processos na cadeia de suprimentos e desempenho do serviço ao cliente: um estudo na indústria calçadista de Franca, Revista Gestão \& Produção, São Carlos, v. 16, n.2, p.232-244, abr.-jun. 
Ho, W., Zheng, T., Yildiz, H., \& Talluri, S. (2015). Supply chain risk management: a literature review, International Journal of Production Research, Vol. 53 No. 16, pp. 5031-5069.

Hung, Y. C., \& Chuang, Y. H. (2009, July). Factors affecting knowledge sharing behavior: A content analysis of empirical findings. In International Conference of Pacific Rim Management

Jaruzelski, B., \& Dehoff, K. (2011). The global innovation 1000: how the top innovators keep winning. Strategy and Business, n. 61, p. 48.

Johnsen, T. (2009). Supplier involvement in new product development and innovation: Taking stock and looking to the future. Journal of Purchasing and Supply Management, v. 15, n. 3, p. 187-197.

Kayakutlu, G., Büyüközkan, G. (2010). Effective supply value chain based on competence success. Supply Chain Management: An International Journal, 15(2), p.129-138.

Kim, B. (2000). Coordinating an innovation in supply chain management. European Journal Of Operational Research, v. 123, n. 3, p. 568-584.

Kyläheiko, K., Jantunen, A., Puumalainen, K., \& Luukka, P. (2011). Value of knowledgeTechnology strategies in different knowledge regimes. International Journal of Production Economics, 131(1), 273-287.

Kyung, T. K, Liang, H., J., \& Seung L. (2016). Impacts of Buyer-Supplier Cooperation on Trust and Performance: Moderating Role of Governance Mechanism. Pesquisa em Ciências da Distribuição, 14, 113-121.

Leonard-Barton, D. (1995). Wellsprings of Knowledge: Building and Sustaining the Sources of Innovation. Boston: Harvard Business School Press.

Mintzberg, H. (1979). The Structure of Organizations. Englewood Cliffs, NJ: Prentice- Hall.

Muniz Jr, J., Maia, F. G. M., \& Viola, G. (2011). Os principais trabalhos na teoria do conhecimento tácito: pesquisa bibliométrica 2000-2011. XIV Simpósio de Administração da Produção, Logística e Operações Internacionais. Anais... São Paulo.

Nonaka, I., \& Takeuchi, H. (1997). Criação de conhecimento na empresa. Rio de Janeiro: Campus.

Porter, M. (1989). Vantagem competitiva: criando e sustentando um desempenho superior. Rio de Janeiro: Elsevier.

Prahalad, C. K., \& Hamel, G. (1990). The core competence ofthe corporation. Harvard Business Review, 3.

Ribeiro, J. S. D. A. N. (2019). Modelo de análise do compartilhamento de conhecimento e inovação no desempenho de entrega: um estudo sobre Integração da Cadeia de Suprimentos. Tese. Programa 
de Pós-Graduação em Sistemas de Informação e Gestão do conhecimento da Universidade FUMEC. Belo Horizonte. Minas Gerais.

Sampieri, R. H., Collado, C. F., \& Lucio, M. D. P. B. (2010). Metodologia de pesquisa.

Simatupang, T. M., Wright, A. C., \& Sridharan, R. (2002). The knowledge of coordination for supply chain integration. Business Process Management Journal, v. 8, n.3, p.289-308.

Sveiby, K. E. (1998). A nova riqueza das organizações: gerenciando e avaliando patrimônios de conhecimento. Rio de Janeiro: Campus.

Szulanski, G. (1996). Exploring Internal Stickness: Impediments to the Transfer of Best Practice Within the Firm. Strategic Management Journal, n. 17, p. 27-43.

Teixeira, T. M. C., \& Valentim, M. L. P. (2017). A contribuição da gestão do conhecimento para o processo de inteligência competitiva organizacional. Anais... XVIII Encontro Nacional de Pesquisa em Ciência da Informação (XVIII ENANCIB).

Tse, Y.K., Matthews, R.L., Tan, K.H., Sato, Y., \& Pongpanich, C. (2016). Unlocking supply chain disruption risk within the thai beverage industry, Industrial Management \& Data Systems, Vol. 116 No. 1, pp. 21-42.

Velu, C. (2014). Business model innovation and third-party alliance on the survival of new firms. Technovation, n. 35, p. 1-11.

Vergara, S. C. (2014). Projetos e Relatórios de Pesquisa em Administração. São Paulo-SP: Editora Atlas S.A.

Yang, J, Yu, G., Liu, M., \& Rui, M. (2016). Improving learning alliance performance for manufacturers: Does knowledge sharing matter? International Journal of Production Economics, Elsevier, vol. 171(P2), p. 301-308. 